\title{
Predictors of Treatment Response to an Adjunctive Emotion Regulation Group Therapy for Deliberate Self-harm among Women with Borderline Personality Disorder
}

\author{
Kim L. Gratz , Katherine L. Dixon-Gordon, and Matthew T. Tull \\ Department of Psychiatry and Human Behavior, University of Mississippi Medical Center
}

\section{Abstract}

Despite evidence for the efficacy of several treatments for deliberate self-harm (DSH) within borderline personality disorder (BPD), predictors of response to these treatments remain unknown. This study examined baseline demographic, clinical, and diagnostic predictors of treatment response to an adjunctive emotion regulation group therapy (ERGT) for DSH among women with BPD. A recent RCT provided evidence for the efficacy of this ERGT (relative to a treatment-asusual only waitlist condition). Participants in this study include the full intent-to-treat sample who began ERGT (across treatment and waitlist conditions; $N=51$ ). Baseline diagnostic and clinical data were collected at the initial assessment, and outcome measures of DSH and self-destructive behaviors, emotion dysregulation/avoidance, and BPD symptoms (among others) were administered at pre-treatment, post-treatment, and 3- and 9-months post-treatment. Notably, both demographic variables and characteristics of participants' ongoing therapy in the community had minimal impact on treatment response. However, several indicators of greater severity in domains relevant to this ERGT (i.e., baseline emotion dysregulation and BPD criteria, lifetime and recent DSH, and past-year hospitalization and suicide attempts) predicted better responses during treatment and follow-up across the primary targets of treatment. Likewise, several co-occurring disorders (i.e., social phobia, panic disorder, and a cluster B personality disorder) predicted greater improvements in BPD symptoms during treatment or follow-up. Finally, although co-occurring generalized anxiety disorder, posttraumatic stress disorder, and cluster A and C personality disorders were associated with poorer treatment response during follow-up, most of these effects reflected a lack of continued improvements during this period (vs. worsening of symptoms).

\section{Keywords}

borderline personality; treatment response; predictors; group therapy; self-injury

Borderline personality disorder (BPD) is a serious mental health problem associated with severe functional impairment, high rates of co-occurring psychiatric disorders, elevated risk for a variety of self-destructive and health-compromising behaviors, and substantial economic, societal, and personal costs (Gunderson, 2001; Skodol et al., 2002; van Asselt,

${ }^{*}$ Correspondence concerning this article should be addressed to Kim L. Gratz, Department of Psychiatry and Human Behavior, University of Mississippi Medical Center, 2500 North State Street, Jackson, MS 39216, USA. Phone: (601) 815-6450; Fax: (601) 984-4489; KLGratz@aol.com. 
Dirksen, Arntz, \& Severens, 2007). One particularly clinically-important behavior commonly associated with BPD (Linehan, 1993) and implicated in the high levels of health care utilization among individuals with BPD (Zanarini, 2009) is deliberate self-harm (DSH; defined as the deliberate, direct, self-inflicted destruction of body tissue without suicidal intent and for purposes not socially sanctioned; Gratz, 2001). Although the past two decades have seen the development of several efficacious treatments for DSH within BPD, including Dialectical Behavior Therapy (DBT; Linehan, 1993; Linehan et al., 2006), MentalizationBased Treatment (Bateman \& Fonagy, 1999, 2004), and an adjunctive emotion regulation group therapy (ERGT; Gratz \& Gunderson, 2006; Gratz, Tull, \& Levy, in press), little is known about the factors that predict response to these treatments. However, given that many patients continue to report considerable difficulties following completion of these treatments, research focused on identifying the patient characteristics associated with better or worse treatment response has great clinical and public health significance. Indeed, according to Kazdin (2001), identification of the predictors of treatment response is critical to the development and effective application of a treatment. Such research has the potential to inform the modification of interventions to facilitate therapeutic change, as well as to identify the particular patients most likely to benefit from a treatment.

Despite the clinical relevance of this area of inquiry, no studies have examined predictors of response to empirically-supported treatments for DSH within BPD, and few have examined predictors of treatment response in empirically-supported treatments for BPD in general (see Black et al., 2009). Moreover, of the studies that have been conducted, most have focused on a narrow set of predictors, limiting the ability to aggregate results across studies. Nonetheless, extant research in this area highlights several potential predictors of treatment response within BPD treatments.

For example, in one of the most comprehensive studies examining predictors of response to a BPD treatment to date, Black et al. (2009) identified a number of significant predictors of treatment response to an adjunctive 20-week cognitive-behavioral group treatment for BPD. In particular, they found that several indices of greater clinical severity at baseline (i.e., BPD symptom severity, global severity, number of co-occurring personality disorders, history of DSH, and lifetime substance use disorder) were associated with greater improvements in BPD symptom severity during both treatment and a one-year follow-up. Similar findings were reported by Yen, Johnson, Costello, and Simpson (2009), who examined baseline BPD criteria as predictors of response during a 3-month period following discharge from a 5-day partial hospitalization DBT program. Specifically, they found that the presence of the BPD criterion of impulsivity predicted greater improvements in self-injurious behaviors (across both DSH and suicidal behaviors) and anger expression; the presence of the BPD criterion of unstable relationships predicted greater improvements in self-injurious behaviors and general psychiatric symptom severity; and the presence of the BPD criterion of emptiness predicted greater improvements in general psychiatric symptom severity, dissociation, and depression symptoms.

Conflicting results have been reported in other studies, however, which found that greater baseline clinical severity predicted worse response to inpatient BPD treatments. For example, baseline severity of dissociation was associated with fewer improvements in 
general psychiatric symptom severity over the course of an inpatient DBT program (Kleindienst et al., 2011). Likewise, several indicators of greater baseline clinical severity, including severity of identity and interpersonal problems (Hull, Clarkin, \& Kakuma, 1993), antisocial personality traits (Clarkin, Hull, Yeomans, Kakuma, \& Cantor, 1994), and cooccurring major depression (Goodman, Hull, Clarkin, \& Yeomans, 1998), were associated with worse response on a measure of general psychiatric symptom severity during a psychodynamic inpatient BPD treatment.

Although the aforementioned studies have yielded mixed results with regard to the particular patient characteristics associated with better or worse response to BPD treatments, this is likely due to differences in the treatments examined across these studies (which vary greatly in setting, duration, intensity, focus, and structure). For example, whereas baseline clinical severity and co-occurring disorders have been found to predict better response to outpatient cognitive-behavioral treatments, they appear to predict worse response to inpatient and/or psychodynamic treatments. Although the implications of a more severe clinical presentation may differ as a function of the level of care (with greater severity in the context of an inpatient setting meaning something different than greater severity in an outpatient setting), some of these differences may also reflect different predictors of treatment response depending on the focus and structure of the treatment. Specifically, the greater structure, more explicit focus on reducing maladaptive behaviors, and stronger emphasis on skills training and outside-of-session assignments in the cognitive-behavioral treatments mentioned above may make these interventions particularly well-suited for more severe and multi-problem patients (who likely exhibit greater behavioral dysregulation). Conversely, a higher level of functioning and greater stability may be needed to benefit fully from a less structured and directive treatment (such as the specific psychodynamic treatments noted above). Indeed, one of the purposes of examining predictors of treatment response is to answer the clinically-important question: what treatments work best for whom? Such research has the potential to identify the particular patients most likely to benefit from any given treatment, as well as those patients for whom a specific treatment may be contraindicated (with findings of relatively few significant predictors of treatment response suggestive of the broader applicability and potential transportability of a treatment). Thus, studies examining a comprehensive set of predictors of treatment response within and across specific treatments for BPD are needed.

The current study sought to extend extant research by providing a comprehensive evaluation of the predictors of treatment response to one empirically-supported treatment for DSH among individuals with BPD: ERGT. Created by Gratz and colleagues (Gratz \& Gunderson, 2006; Gratz \& Tull, 2011) to augment the usual treatments provided by clinicians in the community by directly targeting both DSH and its proposed underlying mechanism of emotion dysregulation (Chapman, Gratz, \& Brown, 2006; Gratz, 2007; Gratz \& Gunderson, 2006; Linehan, 1993), this ERGT was developed with the expectation that teaching selfharming BPD patients more adaptive ways of responding to and regulating their emotions would reduce the frequency of their DSH.

To date, three studies have provided support for the utility of this ERGT in the treatment of DSH within BPD, including an open trial (Gratz \& Tull, 2011) and two randomized 
controlled trials (Gratz \& Gunderson, 2006; Gratz et al., in press). The most recent of these, a larger randomized controlled trial (RCT) and uncontrolled 9-month follow-up (see Gratz et al., in press), provided further evidence for the efficacy of this adjunctive ERGT (relative to a treatment as usual [TAU] only waitlist condition), revealing positive effects of this treatment on both the primary treatment targets (i.e., DSH and other self-destructive behaviors, emotion dysregulation, and BPD symptoms) and other relevant outcomes (i.e., depression and stress symptoms, and overall quality of life) within a conservative intent-totreat (ITT) sample. Moreover, findings from the 9-month follow-up period provide preliminary support for the durability of treatment gains, as all improvements observed from pre- to post-treatment were maintained or further improved upon at follow-up, including additional significant improvements from post-treatment through 9-month follow-up for DSH, emotion dysregulation, experiential avoidance, BPD symptoms, and quality of life (Gratz et al., in press).

The current study examines predictors of treatment response across the primary outcomes of interest (including DSH and other self-destructive behaviors, emotion dysregulation/ avoidance, and BPD symptoms) within the full ITT sample of participants who received ERGT in this RCT. Specifically, we used a piecewise multilevel modeling approach to examine the baseline demographic, clinical, and diagnostic predictors of treatment response to this ERGT during the treatment and follow-up periods. Given the explicit focus of this treatment on reducing behavioral and emotion dysregulation and increasing skill use and generalization (as well as its similarity in treatment structure, intensity, duration, and behavioral orientation to the treatment examined by Black et al. [2009]), we expected that patients with greater clinical severity would experience greater benefits from this treatment. Likewise, given evidence that co-occurring disorders predicted better response to Black et al.'s (2009) cognitive-behavioral treatment, we hypothesized that greater diagnostic severity (in the form of co-occurring disorders) would also predict better responses to this ERGT. Conversely, given evidence for the efficacy of this treatment within a diverse and underserved sample receiving a range of TAU and primarily supportive or dynamic individual therapy (rather than an ERGT-consistent cognitive-behavioral therapy), we did not expect treatment response to vary as a function of participant demographics or characteristics of their TAU (providing further support for the potential applicability and transportability of this treatment).

\section{Method}

\section{Sample and Procedures}

All methods were approved by the institution's Institutional Review Board. Participants were obtained through referrals by clinicians and self-referrals in response to advertisements for an "emotion regulation skills group for women with self-harm" posted online and throughout the community. Inclusion criteria included: (a) threshold or subthreshold diagnosis of BPD (given evidence that even subthreshold BPD is clinically-meaningful; Clifton \& Pilkonis, 2007); (b) a history of repeated DSH, with at least one episode in the past six months; (c) having an individual therapist, psychiatrist, or case manager; and (d) being a woman aged 18-60. To increase generalizability of the sample, exclusion criteria 
included only diagnoses of a primary psychotic disorder, bipolar I disorder, and current (past month) substance dependence. Participants meeting inclusion and exclusion criteria were matched on four prognostic variables (emotion dysregulation, number of lifetime incidents of DSH, global assessment of functioning scores, and age) and randomly assigned by the principal investigator (PI) to either the ERGT + TAU $(n=31)$ or TAU waitlist $(n=30)$ condition using a stratified randomization procedure.

Random assignment to the treatment or waitlist condition occurred as soon as enough participants had been screened; therefore, time between initial assessment and randomization ranged from $<1$ week to approximately 4 months (mean $=29$ days). Five treatment cohorts were recruited from June 2009 to December 2010. Pre-treatment and waitlist assessments were completed within one week prior to the start of the group for participants in the treatment condition; post-treatment and -waitlist assessments were completed within one week following the end of the group. The post-waitlist assessment served as the pre-treatment assessment for participants in the waitlist condition, with their post-treatment assessment occurring within one week after the end of their group. For all participants, follow-up assessments were completed 3- and 9-months (i.e., 14- and 38weeks) following completion of the post-treatment assessment. All assessments were conducted by trained assessors masked to participant condition.

The participants included in the current study include the full ITT sample of participants who received ERGT (across both the treatment and waitlist conditions; $N=51$ ). Table 1 provides demographic, clinical, and diagnostic data on these participants. Detailed information on patient enrollment and disposition across the study is available elsewhere (see Gratz et al., in press).

\section{Measures}

Diagnostic and clinical interviews-The Structured Clinical Interview for DSM-IV Axis I Disorders (SCID-I/P; First, Spitzer, Gibbon, \& Williams, 1996) was used to assess for the exclusion criteria, as well as current and lifetime Axis I disorders. The SCID-I/P has adequate inter-rater and test-retest reliability (Lobbestael, Leurgans, \& Arntz, 2011; Zanarini et al., 2000). The Diagnostic Interview for DSM-IV Personality Disorders (DIPD-IV; Zanarini, Frankenburg, Sickel, \& Young, 1996) was used to assess for the presence of Axis II personality disorders. The DIPD-IV has been found to demonstrate good inter-rater and test-retest reliability for the assessment of personality disorders (Zanarini et al., 2000).

In addition to these diagnostic interviews, the following interviews were administered during the initial assessment to collect baseline clinical data: (a) a modified version of the Lifetime Parasuicide Count (Linehan \& Comtois, 1996), used to assess lifetime suicidal behaviors; (b) an interview version of the Deliberate Self-Harm Inventory (Gratz, 2001), used to assess lifetime DSH; and (c) the Treatment History Interview (THI; Linehan \& Heard, 1987), used to assess past-year psychiatric treatment, including characteristics (e.g., type, intensity, frequency) of participants' ongoing therapy in the community.

All interviews were conducted by clinical assessors trained to reliability with the PI ( $\kappa \geq$ 0.80 ). All interviews were reviewed by the PI; diagnostic discrepancies were found in < 
$10 \%$ of cases. In these instances, areas of disagreement were discussed and a consensus was reached.

Outcome measures-The following measures were administered at pre-treatment, posttreatment, and 3- and 9-months post-treatment to assess changes during treatment and follow-up.

The Deliberate Self-Harm Inventory (DSHI; Gratz, 2001) is a 17-item self-report questionnaire that assesses various aspects of DSH (including frequency) over specified time periods. The DSHI demonstrates adequate test-retest reliability and construct, discriminant, and convergent validity among diverse nonclinical and patient samples (Gratz, 2001; Fliege et al., 2006). A continuous variable measuring frequency of DSH over the specified time period (e.g., in the 3.5 months before the study, since the last assessment) was created by summing participants' scores on the frequency questions for each item $(\alpha=0.75)$.

The Self-Harm Inventory (SHI; Sansone, Wiederman, \& Sansone, 1998) is a 22-item selfreport measure of the presence and frequency of various self-destructive behaviors, including substance abuse, disordered eating behaviors, risky sexual behavior, and suicidal behaviors. The SHI has good convergent and predictive validity (Sansone et al., 1998). This study used a modified version of the SHI to assess past-month frequency of self-destructive behaviors $(a=0.67)$.

The Difficulties in Emotion Regulation Scale (DERS; Gratz \& Roemer, 2004) is a 36-item self-report measure that assesses individuals' typical levels of emotion dysregulation across six domains: emotional nonacceptance, difficulties controlling impulsive behaviors and engaging in goal-directed behaviors when distressed, limited access to effective regulation strategies, and lack of emotional awareness and clarity $(a=0.93$ in this sample). The DERS has good test-retest reliability and construct and predictive validity (Gratz \& Roemer, 2004; Gratz \& Tull, 2010).

The Acceptance and Action Questionnaire (AAQ; Hayes et al., 2004) is a 9-item, self-report measure of experiential avoidance, or the tendency to avoid unwanted internal experiences (particularly emotions). The AAQ has adequate convergent, discriminant, and concurrent validity (Hayes et al., 2004). Higher scores indicate greater experience avoidance $(a=0.75)$.

The Zanarini Rating Scale for Borderline Personality Disorder (ZAN-BPD; Zanarini, 2003) is a clinician-administered instrument for assessing change in BPD symptoms over time. The ZAN-BPD demonstrates good reliability and validity (Zanarini, 2003), and was used to provide an interviewer-based assessment of past-week BPD symptom severity $(a=0.81)$. Interviews were conducted by clinical assessors trained to reliability with the PI (ICC=0.92).

The Borderline Evaluation of Severity over Time (BEST; Pfohl et al., 2009) is a 15-item, self-report measure of past-month BPD symptom severity $(a=0.84$ in this sample). The BEST demonstrates adequate reliability and convergent and discriminant validity (Pfohl et al., 2009). 


\section{Treatment}

Emotion regulation group therapy-This ERGT is based on the conceptualization of emotion regulation as a multidimensional construct involving the: (a) awareness, understanding, and acceptance of emotions; (b) ability to engage in goal-directed behaviors and inhibit impulsive behaviors when experiencing negative emotions; (c) use of situationally-appropriate strategies to modulate the intensity or duration of emotions, rather than to eliminate emotions; and (d) willingness to experience negative emotions as part of pursuing meaningful activities in life (Gratz \& Roemer, 2004). ERGT draws from two acceptance-based behavioral therapies, Acceptance and Commitment Therapy (Hayes, Strosahl, \& Wilson, 1999) and DBT (Linehan, 1993), and emphasizes the following themes: (a) the potentially paradoxical effects of emotional avoidance, (b) the emotion-regulating consequences of emotional acceptance and willingness, and (c) the importance of controlling behaviors when emotions are present, rather than controlling emotions themselves. A detailed manual has been developed and a full description of the specific topics addressed each week is available elsewhere (Gratz \& Gunderson, 2006). Groups meet weekly for 90 minutes over 14 weeks and are limited to 6 patients per group. Treatment integrity data for this RCT are available elsewhere (see Gratz et al., in press).

Treatment as usual-All participants were required to have an individual clinician, and all continued with their ongoing outpatient treatment over the course of the RCT.

Participants had been meeting with their individual clinicians for an average of 15.5 months $(\mathrm{SD}=21.1$; range $=1$ month to 9 years) prior to the start of the study, with $76 \%$ reporting a duration of $\geq 2$ months. Few participants (23\%) received group therapy outside of this ERGT, and 57\% received less than one hour of individual therapy per week. Further information on participants' TAU is provided in Table 1. With regard to the individual clinicians of participants, $20 \%$ were in private practice and the others worked in a community mental health center (43\%), college counseling center (12\%), or hospital (24\%). In regard to their training, $69 \%$ had a master's degree, $22 \%$ were clinical psychologists, and $10 \%$ were psychiatrists. Most participants (>60\%) were receiving supportive or dynamic individual therapy (according to the THI and discussions with clinicians); however, 19.6\% were receiving cognitive-behavioral therapy (although not DBT).

\section{Analysis Plan}

Given the hierarchical structure of our data, in which repeated measures ratings (Level 1) are nested within participants (Level 2), we used a multilevel modeling approach to examine baseline predictors of response during the treatment and follow-up periods (as evidenced by significant time by predictor interactions; Longford, 1993). Specifically, Level 1 data comprised outcomes rated over time (pre-treatment, post-treatment, 3-month follow-up, and 9-month follow-up), and Level 2 data include baseline predictors of treatment response (across demographic, clinical, and diagnostic characteristics). Such multilevel models confer an advantage for studies relying on repeated measures, as participants with missing values can contribute to the estimation of the residual error and random intercept terms (Krueger \& Tian, 2004), resulting in an ITT sample included in all analyses. Because the trajectory of the outcome variables across time was not expected to be linear, a piecewise linear model was created with the pre-treatment assessment serving as the reference point for the first 
linear time variable (Time 1; with the post-treatment assessment occurring 14 weeks later) and a second linear time variable commencing at post-treatment (Time 2; influencing the 3month and 9-month follow-ups that occur 14 and 38 weeks post-treatment, respectively) (Heck, Thomas, \& Tabata, 2010). Models included fixed effects of Time 1, Time 2, and each predictor, as well as the Time $1 \times$ predictor and Time $2 \times$ predictor interactions.

Prior to conducting primary analyses, all continuous predictor variables (with the exception of age) were grand-mean centered. In order to model DSH over time in light of the unequal intervals between assessments, DSH frequencies were scaled to be the frequency per 14 weeks. Null models were examined, and the variance components for the intercept of each of these models were significant, suggesting significant differences across individuals. In each model, the residual for the intercept was permitted to vary across individuals, and the residuals for the slopes of time were permitted to vary if the slope was significant when examining the variance components in the null models. Next, covariance structures were determined by comparing the model fit criteria (e.g., Akaiki information criteria), and models were estimated using restricted estimated maximum likelihood (Figuerdo \& Olderbak, 2008). We followed up significant interactions using simple slopes tests for multilevel models (Preacher, Curran, \& Bauer, 2006). Slopes are reported at both levels of the moderator for dichotomous variables, and $1 \mathrm{SD}$ above and below the mean for continuous variables. Given our small sample size (and related risk for Type II error; Raudenbush et al., 2011), we did not apply an alpha correction to our analyses (see Tutzauer, 2003).

\section{Results}

Descriptive statistics for all outcome measures across all time points are presented in a previous manuscript (Gratz et al., in press). Results of the multilevel models for the primary outcomes of interest (including DSH, self-destructive behaviors, emotion dysregulation, experiential avoidance, and BPD symptoms) are presented in Table 2. All final models demonstrated significant improvement compared to the null (i.e., only intercept included) maximum likelihood-estimated models in terms of the deviance statistics from each model, distributed as chi-square with degrees of freedom equal to the difference in parameters estimated across the models.

\section{Demographic Characteristics}

Demographic characteristics had minimal impact on treatment response, and none of these characteristics predicted treatment response across the primary treatment targets (i.e., DSH, emotion dysregulation, or BPD symptoms). However, age, education, and income predicted treatment response on the measure of experiential avoidance, with greater improvements in experiential avoidance during treatment found among younger participants, $b=-52, \mathrm{SE}=$. $11, p<.001$ (relative to older participants, $b=-.21, \mathrm{SE}=.10, p=.04$ ), and participants with a high school education, $b=-.51, \mathrm{SE}=.09, p<.001$ (vs. college education or higher, $b=-$. $06, \mathrm{SE}=0.12, p=.60$ ), and continued improvements in experiential avoidance during the follow-up period found among participants with higher incomes, $b=-.24, \mathrm{SE}=.07, p<$. 001 (vs. those with lower incomes, whose levels of experiential avoidance remained stable 
during follow-up; $b=.06, \mathrm{SE}=.08, p=.45)$. When these demographic predictors were examined simultaneously, both high school (vs. college) education and higher income continued to predict greater improvements in experiential avoidance during treatment and follow-up, respectively (time $\times$ predictor parameters $=.37$ and .35 , SEs $=.17$ and $.12, p s<$. 05 ), whereas age did not remain a significant predictor (time $\times$ predictor parameter $=.01, \mathrm{SE}$ $=.01, p=.15)$.

\section{Clinical Characteristics}

Characteristics of participants' ongoing therapy in the community also had little impact on treatment response, with only one effect emerging as significant. Specifically, a greater intensity of overall TAU was associated with fewer improvements in BPD symptoms during treatment, $b=-.13, \mathrm{SE}=.12, p=.28$ (relative to participants with less TAU; $b=-.59$, SE $=.11, p<.001)$.

With regard to other clinical characteristics, several indicators of greater baseline severity in domains relevant to this ERGT were found to predict better responses during treatment and follow-up. Specifically, higher levels of baseline emotion dysregulation and the presence of more BPD criteria on the DIPD-IV predicted greater improvements in BPD symptoms over the course of treatment, $b \mathrm{~s}=-.68$, SEs $=.09, p \mathrm{~s}<.001$ (relative to participants with lower baseline emotion dysregulation and fewer BPD criteria; $b \mathrm{~s}=-.38$, SEs $\geq .08, p \mathrm{~s}<.001$ ). Likewise, greater lifetime and past 3-month DSH frequency predicted greater continued improvements in DSH during the follow-up period, $b \mathrm{~s}=-.03$, SEs $=.004, p \mathrm{~s}<.01$ (relative to participants with lower levels of baseline DSH; $b s=-.01$, SEs $=.004, p s<.05$ ). Moreover, a past-year inpatient hospitalization and suicide attempt predicted greater improvements in experiential avoidance, with a past-year hospitalization predicting greater improvements in experiential avoidance during treatment, $b=-.70, \mathrm{SE}=.20, p<.001$ (relative to non-hospitalized participants; $b=-.27, \mathrm{SE}=.08, p=.001$ ), and a suicide attempt in the past year predicting greater continued improvements in experiential avoidance during the follow-up period, $b=-.36, \mathrm{SE}=.13, p=.007$ (relative to participants without a pastyear suicide attempt, whose levels of experiential avoidance remained stable during followup; $b=-.05, \mathrm{SE}=.06, p=.37$ ).

Nonetheless, despite the general tendency for greater baseline severity to predict better treatment response, as well as findings that greater lifetime and past 3-month DSH predicted greater continued improvements in DSH during follow-up, baseline DSH severity (as indexed by greater lifetime and past 3-month DSH frequency) was associated with poorer treatment response on the self-report measure of BPD symptoms (although not the clinicianadministered measure) during follow-up, such that participants with greater lifetime DSH reported an increase in BPD symptoms during the follow-up $(b=14.22, \mathrm{SE}=5.91, p=.02)$ and those with greater past 3-month DSH reported an absence of further improvements in BPD symptoms during follow-up ( $b=-.06, \mathrm{SE}=.08, p=.45$ ), compared to participants with lower levels of baseline DSH (who exhibited continued improvements in BPD symptoms during the follow-up; $b s=-.33$ and -.32 , SEs $=.09, p s<.001$ ). Moreover, one indicator of clinical severity (past-year inpatient hospitalization) predicted fewer 
improvements in DSH over the course of treatment, $b=-.01, \mathrm{SE}=.01, p=.35$ (relative to non-hospitalized participants; $b=-.04, \mathrm{SE}=.01, p<.001$ ).

\section{Diagnostic Characteristics}

Co-occurring disorders also predicted treatment response. Consistent with hypotheses, the presence of co-occurring social phobia was associated with better responses during treatment, including greater improvements in BPD symptoms $(b=-.87, \mathrm{SE}=.23, p<.001)$ and self-destructive behaviors $(b=-3.94, \mathrm{SE}=1.11, p<.001)$ relative to participants without social phobia (for BPD symptoms: $b=-.26, \mathrm{SE}=.11, p=.02$; for self-destructive behaviors: $b=-1.12, \mathrm{SE}=.56, p=.047)$. Likewise, the presence of a co-occurring cluster $\mathrm{C}$ disorder predicted greater improvements in self-destructive behaviors during treatment, $b=$ $-3.35, \mathrm{SE}=.88, p<.001$ (relative to participants without a cluster $\mathrm{C}$ disorder; $b=-.95$, SE $=.63, p=.14)^{1}$ and the presence of a co-occurring cluster B disorder predicted continued improvements in BPD symptoms during follow-up, $b=-.16, \mathrm{SE}=.06, p=.01$ (vs. participants without a co-occurring cluster B disorder, whose BPD symptoms remained stable during follow-up, $b=.002, \mathrm{SE}=.02, p=.94$ ). Finally, co-occurring panic disorder was associated with greater improvements in BPD symptoms during treatment, $b=-.98$, SE $=.12, p<.001$ (relative to participants without this disorder; $b=-.40, \mathrm{SE}=.07, p<.001$ ), although the presence of this disorder was also associated with an increase in selfdestructive behaviors during follow-up, $b=1.06, \mathrm{SE}=.39, p=.01$ (vs. participants without panic disorder, whose self-destructive behaviors remained stable during follow-up; $b=-.15$, $\mathrm{SE}=.21, p=.47$ ) and an absence of further improvements in BPD symptoms during followup, $b=.06, \mathrm{SE}=.05, p=.20$ (vs. participants without panic disorder, whose BPD symptoms continued to improve during follow-up; $b=-.06, \mathrm{SE}=.03, p=.04$ ). The effects of both panic disorder and a co-occurring cluster B personality disorder on improvements in BPD symptoms during treatment and follow-up, respectively, remained significant when examining these diagnostic predictors simultaneously (time $\times$ predictor parameters $=-.56$ and -.17 , SEs $=.14$ and $.06, p s<.05)$.

Notably, although other co-occurring disorders were associated with poorer treatment response, only one predicted poorer response across the primary treatment targets (i.e., DSH, emotion dysregulation, or BPD symptoms). Further, all of these effects emerged during the follow-up period and most reflected a lack of continued improvements during this period (rather than a worsening of symptoms). In particular, co-occurring generalized anxiety disorder (GAD) was associated with an absence of continued improvements in both DSH ( $b$ $=-.004, \mathrm{SE}=.01, p=.52)$ and experiential avoidance $(b=.17, \mathrm{SE}=.12, p=.18)$ during the follow-up (vs. participants without GAD, who evidenced continued improvements in these outcomes during follow-up; for DSH: $b=-.02, \mathrm{SE}=.003, p<.001$; for experiential avoidance: $b=-.17, \mathrm{SE}=.06, p=.004)$. Likewise, the presence of co-occurring posttraumatic stress disorder (PTSD) or a cluster A or C personality disorder was associated with an absence of continued improvements in experiential avoidance during the follow-up (for PTSD: $b=.07, \mathrm{SE}=.10, p=.48$; for cluster $\mathrm{A}: b=.40, \mathrm{SE}=.23, p=.09$; for cluster C:

\footnotetext{
${ }^{1}$ When social phobia and a cluster $\mathrm{C}$ personality disorder were examined simultaneously as predictors of improvements in selfdestructive behaviors during treatment (along with panic disorder and a cluster A personality disorder, which were associated with poorer response on this outcome during follow-up), both approached significance as unique predictors ( $p s=.06$ and .07 , respectively).
} 
$b=.10, \mathrm{SE}=.09, p=.26$ ), compared to participants without these disorders (who evidenced continued improvements in experiential avoidance during follow-up; $b \mathrm{~s}=-.19,-.14$, and - . 23 , SEs $=.07, .06$, and $.06, p s \leq .01$ ). When these diagnostic predictors of poorer response on the measure of experiential avoidance were examined simultaneously, only a cooccurring cluster $\mathrm{C}$ personality disorder remained a significant predictor (time $\times$ predictor parameter $=.23, \mathrm{SE}=.11, p=.04)$; the other disorders were not significant unique predictors of poorer response on this outcome ( $p s>.23$ ). Finally, the presence of a cooccurring cluster A personality disorder was associated with an increase in self-destructive behaviors during follow-up, $b=2.31, \mathrm{SE}=.63, p<.001$ (relative to participants without a co-occurring cluster A disorder, whose self-destructive behaviors remained stable during follow-up; $b=.08, \mathrm{SE}=.17, p=.63$ ). Notably, the negative effects of both a cluster A personality disorder and panic disorder on self-destructive behaviors during follow-up remained significant when examining all significant diagnostic predictors of treatment response on this measure simultaneously (time $\times$ predictor parameters $=2.40$ and 1.06 , SEs $=.70$ and .39 , respectively; $p$ s $<.01$ ).

\section{Discussion}

Despite increasing evidence for the efficacy of several treatments for DSH among patients with BPD, the predictors of response to these treatments remain unknown, thereby limiting our understanding of the patients who may be more or less likely to benefit from these interventions. This area of inquiry has important research and clinical implications, aiding in the identification of appropriate inclusion and exclusion criteria for future clinical trials and facilitating treatment decision making for particular patients (Kraemer, Wilson, Fairburn, \& Agras, 2002). Indeed, experts in treatment outcome research have emphasized the importance of examining moderators of treatment outcomes in order to guide treatment development and refinement and clinical decision making (Kazdin, 2001; Kazdin \& Weisz, 1998; Kraemer et al., 2002). Thus, in line with these recommendations, the current exploratory study examined a wide range of demographic, clinical, and diagnostic predictors of treatment response to an adjunctive ERGT with demonstrated efficacy in the treatment of DSH within BPD (Gratz \& Gunderson, 2006; Gratz et al., in press). Findings of this study speak to the potential applicability and transportability of this ERGT and highlight the particular patients likely to experience the greatest benefits from this treatment.

Overall, one of the most notable findings is the relative absence of significant predictors of response to this brief treatment. Indeed, despite examining a wide range of patient characteristics that could potentially influence treatment response, results revealed relatively few significant predictors of response to this ERGT (particularly during the treatment). These findings provide further support for the transportability of this treatment and its utility across a wide range of BPD patients. Of particular importance, both demographic variables and characteristics of participants' ongoing therapy in the community had minimal impact on treatment response. Specifically, results suggest that the overall utility of this treatment is not dependent on a patient's age, racial/ethnic background, or socioeconomic status. Increasing confidence in the broad applicability of this treatment, these demographic predictors were examined within a socioeconomically and ethnically diverse sample. Likewise, results of this study provide further evidence that the utility of this adjunctive 
ERGT is not dependent on its pairing with a theoretically-similar individual therapy, as having a cognitive-behavioral (vs. non-cognitive behavioral) individual therapist did not influence treatment response on any outcome measure. Treatment response also largely did not vary as a function of the intensity of participants' ongoing therapy in the community (overall or with regard to individual therapy in particular). Together, these findings highlight the potential generalizability of this ERGT, suggesting that this treatment could be offered to a wide variety of individuals in different settings with little concern that its effectiveness would be diminished.

Despite the general pattern of findings supporting the relatively broad applicability of this ERGT, results also suggest a number of clinical and diagnostic characteristics that predict favorable responses to the primary outcomes of interest within this treatment. In particular, and consistent with hypotheses, several indicators of greater baseline clinical severity in domains relevant to this ERGT (including higher levels of baseline emotion dysregulation, the presence of more BPD criteria, greater baseline DSH, and a past-year inpatient hospitalization and suicide attempt) were found to predict better responses during treatment and follow-up on measures of BPD symptoms, DSH, and experiential avoidance. Notably, these findings suggest that the difficulties that likely prompt patients to enter this treatment in no way interfere with its effectiveness; rather, patients with more severe difficulties in these areas appear to benefit more from this brief ERGT (consistent with past findings of a positive association between clinical severity and treatment response in other cognitivebehavioral treatments; e.g., prolonged exposure and cognitive processing therapy for PTSD; Rizvi, Vogt, \& Resick, 2009). Likewise, several co-occurring disorders (including social phobia, panic disorder, and a cluster B personality disorder) predicted greater improvements in BPD symptoms during treatment or follow-up. Given evidence of shared features between each of these disorders and BPD (including behavioral and emotional avoidance and impulsive behaviors in social phobia [Buckner et al., 2009; Hofmann \& Barlow, 2002; Turk, Heimberg, Luterek, Mennin, \& Fresco, 2005]; deficits in interpersonal functioning, anger modulation, and emotional clarity in panic disorder [Carter, Turovsky, \& Barlow, 1994; Moscovitch, McCabe, Antony, Rocca, \& Swinson, 2008; Tull \& Roemer, 2007]; and interpersonal and emotional dysfunction in other cluster B personality disorders [Miller, Campbell, \& Pilkonis, 2007; Russ, Shedler, Bradley, \& Westen, 2008]), it is possible that certain components of this ERGT (e.g., the focus on emotional willingness and valued actions, impulse control strategies, and emotional awareness and clarity) are particularly helpful in reducing these symptoms among BPD patients with these co-occurring disorders.

Finally, although some diagnostic characteristics were found to predict poorer treatment response during the follow-up period, most of these effects reflected a lack of continued improvements during this period rather than a worsening of symptoms. Thus, just as these findings provide further support for the utility of this treatment across a wide range of BPD patients with and without co-occurring psychopathology, they also reveal a subset of BPD patients whose positive responses to treatment may be more difficult to maintain after completion of treatment (and who may benefit from additional interventions or services following completion of this brief ERGT). In particular, findings suggest that co-occurring PTSD, GAD, and cluster A and C personality disorders may impede further progress following completion of this treatment. Findings that patients with co-occurring PTSD, 
$\mathrm{GAD}$, or a cluster $\mathrm{C}$ personality disorder may have greater difficulties maintaining some gains following completion of treatment are consistent with evidence that PTSD and GAD are associated with worse clinical outcomes than the other anxiety disorders (Marciniak et al., 2005; Roemer, Orsillo, \& Barlow, 2002; van Minnen, Arntz, \& Keijsers, 2002), as well as past research suggesting that the co-occurrence of PTSD or a cluster C personality disorder with BPD is associated with worse outcomes and prognosis (Pagura et al., 2010; Shea et al., 2004; Zanarini, Frankenburg, Hennen, Reich, \& Silk, 2006; Zanarini et al., 2004). Likewise, findings that a co-occurring cluster A personality disorder predicted worse outcomes during follow-up are consistent with evidence that schizoid personality traits predict worse response to intensive outpatient DBT (Perroud, Uher, Dieben, Nicastro, \& Huguelet, 2010) and patients with schizotypal personality disorder continue to exhibit significant impairment in functioning following treatment (Mehlum et al., 1991).

Several limitations warrant mention. First, the exploratory nature of this study and relatively large number of analyses (compared to the modest sample size) increases the risk for Type I error; thus, the findings presented should be considered preliminary and in need of further investigation and replication in future clinical trials. Second, although assessments included both clinician-administered and self-report measures, future studies would benefit from the inclusion of behavioral measures of emotion dysregulation and interpersonal functioning as well (e.g., Bray, Barrowclough, \& Lobban, 2007; Gratz, Rosenthal, Tull, Lejuez, \& Gunderson, 2006; Thayer \& Lane, 2000). Third, given our exclusive focus on women with BPD, the generalizability of these findings to adolescents and men remains unclear. Finally, although findings highlight several patient characteristics that may influence treatment response across a 9-month follow-up, longer follow-up periods are needed.

Despite limitations, the present study extends extant literature on the predictors of response to treatments for DSH among individuals with $\mathrm{BPD}$, examining a wide range of predictors across multiple domains (including demographic, clinical, and diagnostic characteristics). Future research is needed to examine other characteristics that may influence response to this ERGT, including personality traits and other relevant individual difference characteristics (see, e.g., Lenzenweger, Clarkin, Levy, Yeomans, \& Kernberg, 2012; Hollander, Swann, Coccaro, Jiang, \& Smith, 2005), the therapeutic alliance and other aspects of the treatment relationship, and motivation for treatment (e.g., Morey, Lowmaster, \& Hopwood, 2010). The consistency of predictors of treatment response across different efficacious treatments for DSH within BPD (e.g., ERGT, DBT, mentalization-based treatment) also warrants investigation. Such research has the potential to identify the particular patients most likely to benefit from various treatment options, thereby providing important information on client-therapy match.

\section{Acknowledgments}

This research was supported by National Institute of Mental Health Grant R34 MH079248, awarded to the first author (KLG). 


\section{References}

Bateman A, Fonagy P. Effectiveness of partial hospitalization in the treatment of borderline personality disorder: A randomized controlled trial. American Journal of Psychiatry. 1999; 156:1563-1569. [PubMed: 10518167]

Bateman AW, Fonagy P. Mentalization-based treatment of BPD. Journal of Personality Disorders. 2004; 18:36-51. [PubMed: 15061343]

Black DW, Allen J, St John D, Pfohl B, McCormick B, Blum N. Predictors of response to systems training for emotional predictability and problem solving (STEPPS) for borderline personality disorder: An exploratory study. Acta Psychiatrica Scandinavica. 2009; 120:53-61. [PubMed: 19183126]

Bray S, Barrowclough C, Lobban F. The social problem-solving abilities of people with borderline personality disorder. Behaviour Research and Therapy. 2007; 45:1409-1417. [PubMed: 16919235]

Buckner JD, Schmidt NB, Lang AR, Small JW, Schlauch RC, Lewinsohn PM. Specificity of social anxiety disorder as a risk factor for alcohol and cannabis dependence. Journal of Psychiatric Research. 2009; 42:230-239. [PubMed: 17320907]

Carter MM, Turovsky J, Barlow DH. Interpersonal relationships in panic disorder with agoraphobia: A review of empirical evidence. Clinical Psychology: Science and Practice. 1994; 1:25-34.

Chapman AL, Gratz KL, Brown MZ. Solving the puzzle of deliberate self-harm: The experiential avoidance model. Behaviour Research and Therapy. 2006; 44:371-394. [PubMed: 16446150]

Clarkin JF, Hull J, Yeomans F, Kakuma T, Cantor J. Antisocial traits as modifiers of treatment response in borderline inpatients. The Journal of Psychotherapy Practice and Research. 1994; 3:307-312. [PubMed: 22700198]

Clifton A, Pilkonis PA. Evidence for a single latent class of Diagnostic and Statistical Manual of Mental Disorders borderline personality pathology. Comprehensive Psychiatry. 2007; 48:70-78. [PubMed: 17145285]

Figuerdo AJ, Olderbak SG. Generalizability theory analysis for psycholinguistic applications. Journal of Memory and Language. 2008; 59:426-433.

First, MB.; Spitzer, RL.; Gibbon, M.; Williams, JBW. Structured clinical interview for DSM-IV axis I disorders - patient edition (SCID-I/P, version 2.0). New York, NY: New York State Psychiatric Institute; 1996.

Fliege H, Kocalevent R, Walter OB, Beck S, Gratz KL, Gutierrez PM, Klapp BF. Three assessment tools for deliberate self-harm and suicide behavior: Evaluation and psychopathological correlates. Journal of Psychosomatic Research. 2006; 61:113-121. [PubMed: 16813853]

Goodman G, Hull JW, Clarkin JF, Yeomans FE. Comorbid mood disorders as modifiers of treatment response among inpatients with borderline personality disorder. The Journal of Nervous and Mental Disease. 1998; 186:616-622. [PubMed: 9788638]

Gratz KL. Measurement of deliberate self-harm: Preliminary data on the Deliberate Self-Harm Inventory. Journal of Psychopathology and Behavioral Assessment. 2001; 23:253-263.

Gratz KL. Targeting emotion dysregulation in the treatment of self-injury. Journal of Clinical Psychology. 2007; 63:1091-1103. [PubMed: 17932982]

Gratz KL, Gunderson JG. Preliminary data on an acceptance-based emotion regulation group intervention for deliberate self-harm among women with borderline personality disorder. Behavior Therapy. 2006; 37:25-35. [PubMed: 16942958]

Gratz KL, Roemer L. Multidimensional assessment of emotion regulation and dysregulation: Development, factor structure, and initial validation of the difficulties in emotion regulation scale. Journal of Psychopathology \& Behavioral Assessment. 2004; 26:41-54.

Gratz KL, Rosenthal MZ, Tull MT, Lejuez CW, Gunderson JG. An experimental investigation of emotion dysregulation in borderline personality disorder. Journal of Abnormal Psychology. 2006; 115:850-855. [PubMed: 17100543]

Gratz, KL.; Tull, MT. Emotion regulation as a mechanism of change in acceptance-and mindfulnessbased treatments. In: Baer, R., editor. Assessing mindfulness and acceptance: Illuminating the process of change. Oakland, CA: New Harbinger Publications; 2010. p. 105-133. 
Gratz KL, Tull MT. Extending research on the utility of an adjunctive emotion regulation group therapy for deliberate self-harm among women with borderline personality pathology. Personality Disorders: Theory, Research, and Treatment. 2011; 2:316-326.

Gratz KL, Tull MT, Levy RL. Randomized controlled trial and uncontrolled 9-month follow-up of an adjunctive emotion regulation group therapy for deliberate self-harm among women with borderline personality pathology. Psychological Medicine. (in press).

Gunderson, JG. Borderline personality disorder: A clinical guide. Washington, DC: American Psychiatric Press; 2001.

Harned MS, Rizvi SL, Linehan MM. Impact of co-occurring posttraumatic stress disorder on suicidal women with borderline personality disorder. American Journal of Psychiatry. 2010; 167:1210 1217. [PubMed: 20810470]

Hayes, SC.; Strosahl, KD.; Wilson, KG. Acceptance and commitment therapy: An experiential approach to behavior change. New York: Guilford Press; 1999.

Hayes SC, Strosahl K, Wilson KG, Bissett RT, Pistorello J, Toarmino D, McCurry SM. Measuring experiential avoidance: A preliminary test of a working model. The Psychological Record. 2004; 54:553-578.

Heck, RH.; Thomas, SL.; Tabata, LN. Multilevel and longitudinal modeling with IBM SPSS. London: Taylor \& Francis Group; 2010.

Hofmann, SG.; Barlow, DH. Social phobia (social anxiety disorder). In: Barlow, DH., editor. Anxiety and its disorders: The nature and treatment of anxiety and panic. 2. New York, NY: Guilford Press; 2002. p. 454-476.

Hollander E, Swann AC, Coccaro EF, Jiang P, Smith TB. Impact of trait impulsivity and state aggression on divalproex versus placebo response in borderline personality disorder. American Journal of Psychiatry. 2005; 162:621-624. [PubMed: 15741486]

Hull JW, Clarkin JF, Kakuma T. Treatment response of borderline inpatients: A growth curve analysis. The Journal of Nervous and Mental Disease. 1993; 181:503-508. [PubMed: 8360641]

Kazdin AE. Progression of therapy research and clinical application of treatment require better understanding of the change process. Clinical Psychology: Science and Practice. 2001; 8:143-151.

Kazdin AE, Weisz JR. Identifying and developing empirically supported child and adolescent treatments. Journal of Consulting and Clinical Psychology. 1998; 66:19-36. [PubMed: 9489260]

Kleindienst N, Limberger MF, Ebner-Priemer UW, Keibel-Mauchnik J, Dyer A, Berger M, Bohus M. Dissociation predicts poor response to dialectial behavioral therapy in female patients with borderline personality disorder. Journal of Personality Disorders. 2011; 25:432-447. [PubMed: 21838560]

Kraemer HC, Wilson GT, Fairburn CG, Agras WS. Mediators and moderators of treatment effects in randomized clinical trials. Archives of General Psychiatry. 2002; 59:877-883. [PubMed: 12365874]

Krueger C, Tian L. A comparison of the general linear mixed model and repeated measures ANOVA using a dataset with multiple missing data points. Biological Research for Nursing. 2004; 6:151157. [PubMed: 15388912]

Lenzenweger MF, Clarkin JF, Levy KN, Yeomans FE, Kernberg OF. Predicting domains and rates of change in borderline personality disorder. Personality Disorders: Theory, Research, and Treatment. 2012; 3:185-195.

Linehan, MM. Cognitive behavioral treatment of borderline personality disorder. New York, NY: Guilford Press; 1993.

Linehan, MM.; Comtois, K. Unpublished measure. University of Washington; Seattle, WA: 1996. Lifetime parasuicidal history.

Linehan MM, Comtois KA, Murray AM, Brown MZ, Gallop RJ, Heard HL, Lindenboim N. Two-year randomized controlled trial and follow-up of dialectical behavior therapy vs therapy by experts for suicidal behaviors and borderline personality disorder. Archives of General Psychiatry. 2006; 63:757-766. [PubMed: 16818865]

Linehan, MM.; Heard, HL. Unpublished measure. University of Washington; Seattle, WA: 1987. Treatment history interview (THI). 
Lobbestael J, Leurgans M, Arntz A. Inter-rater reliability of the structured clinical interview for DSMIV axis I disorders (SCID I) and axis II disorders (SCID II). Clinical Psychology \& Psychotherapy. 2011; 18:75-79. [PubMed: 20309842]

Longford NT. Regression analysis of multilevel data with measurement error. British Journal of Mathematical and Statistical Psychology. 1993; 46:301-311.

Marciniak MD, Lage MJ, Dunayevich E, Russell JM, Bowman L, Landbloom RP, Levine LR. The cost of treating anxiety: The medical and demographic correlates that impact total medical costs. Depression and Anxiety. 2005; 21:178-184. [PubMed: 16075454]

Mehlum L, Friis S, Irion T, Johns S, Karterud S, Vaglum P, Vaglum S. Personality disorders 2-5 years after treatment: A prospective follow-up study. Acta Psychiatrica Scandinavica. 1991; 84:72-77. [PubMed: 1927569]

Miller JD, Campbell WK, Pilkonis PA. Narcissistic personality disorder: Relations with distress and functional impairment. Comprehensive Psychiatry. 2007; 48:170-177. [PubMed: 17292708]

Morey LC, Lowmaster SE, Hopwood CJ. A pilot study of Manual-Assisted Cognitive Therapy with a Therapeutic Assessment augmentation for borderline personality disorder. Psychiatry Research. 2010; 178:531-535. [PubMed: 20537722]

Moscovitch DA, McCabe RE, Antony MM, Rocca L, Swinson RP. Anger experience and expression across the anxiety disorders. Depression and Anxiety. 2008; 25:107-113. [PubMed: 17311254]

Pagura J, Stein MB, Bolton JM, Cox BJ, Grant B, Sareen J. Comorbidity of borderline personality disorder and posttraumatic stress disorder in the US population. Journal of Psychiatric Research. 2010; 44:1190. [PubMed: 20537660]

Perroud N, Uher R, Dieben K, Nicastro R, Huguelet P. Predictors of response and drop-out during intensive dialectical behavior therapy. Journal of Personality Disorders. 2010; 24:634-650. [PubMed: 20958172]

Pfohl B, Blum N, St John D, McCormick B, Allen J, Black DW. Reliability and validity of the borderline evaluation of severity over time (BEST): A self-rated scale to measure severity and change in persons with borderline personality disorder. Journal of Personality Disorders. 2009; 23:281-293. [PubMed: 19538082]

Preacher KJ, Curran PJ, Bauer DJ. Computational tools for probing interaction effects in multiple linear regression, multilevel modeling, and latent curve analysis. Journal of Educational and Behavioral Statistics. 2006; 31:437-448.

Raudenbush, SW.; Spybrook, J.; Congdon, R.; Liu, X.; Martinez, A.; Bloom, H.; Hill, C. Optimal Design Plus Empirical Evidence (Version 3.0). 2011. [Computer software]. Available from www.wtgrantfoundation.org

Rizvi SL, Vogt DW, Resick PA. Cognitive and affective predictors of treatment outcome in cognitive processing therapy and prolonged exposure for posttraumatic stress disorder. Behaviour Research and Therapy. 2009; 47:737-743. [PubMed: 19595295]

Roemer, L.; Orsillo, SM.; Barlow, DH. Generalized anxiety disorder. In: Barlow, DH., editor. Anxiety and its disorders: The nature and treatment of anxiety and panic. 2. New York, NY: Guilford Press; 2002. p. 477-515.

Russ E, Shedler J, Bradley R, Westen D. Refining the construct of narcissistic personality disorder: Diagnostic criteria and subtypes. American Journal of Psychiatry. 2008; 165:1473-1481. [PubMed: 18708489]

Sansone RA, Wiederman MW, Sansone LA. The Self-Harm Inventory (SHI): Development of a scale for identifying self-destructive behaviors and borderline personality disorder. Journal of Clinical Psychology. 1998; 54:973-983. [PubMed: 9811134]

Shea MT, Stout RL, Yen S, Pagano ME, Skodol AE, Morey LC, Zanarini MC. Associations in the course of personality disorders and axis I disorders over time. Journal of Abnormal Psychology. 2004; 113:499-508. [PubMed: 15535783]

Skodol AE, Gunderson JG, McGlashan TH, Dyck IR, Stout RL, Bender DS, Oldham JM. Functional impairment in patients with schizotypal, borderline, avoidant, and obsessive-compulsive personality disorder. American Journal of Psychiatry. 2002; 159:276-283. [PubMed: 11823271]

Thayer JF, Lane RD. A model of neurovisceral integration in emotion regulation and dysregulation. Journal of Affective Disorders. 2000; 61:201-216. [PubMed: 11163422] 
Tull MT, Roemer L. Emotion regulation difficulties associated with the experience of uncued panic attacks: Evidence of experiential avoidance, emotional non-acceptance, and decreased emotional clarity. Behavior Therapy. 2007; 38:378-391. [PubMed: 18021952]

Turk CL, Heimberg RG, Luterek JA, Mennin DS, Fresco DM. Emotion dysregulation in generalized anxiety disorder: A comparison with social anxiety disorder. Cognitive Therapy and Research. 2005; 29:89-106.

Tutzauer F. On the sensible application of familywise alpha adjustment. Human Communication Research. 2003; 29:455-463.

van Asselt ADI, Dirksen CD, Arntz A, Severens JL. The cost of borderline personality disorder: Societal cost of illness in BPD-patients. European Psychiatry. 2007; 22:354-361. [PubMed: 17544636]

van Minnen A, Arntz A, Keijsers GPJ. Prolonged exposure in patients with chronic PTSD: Predictors of treatment outcome and dropout. Behaviour Research and Therapy. 2002; 40:439-457. [PubMed: 12002900]

Yen S, Johnson J, Costello E, Simpson EB. A 5-day dialectical behavior therapy partial hospital program for women with borderline personality disorder: Predictors of outcome from a 3-month follow-up study. Journal of Psychiatric Practice. 2009; 15:173-182. [PubMed: 19461390]

Zanarini MC. Zanarini rating scale for borderline personality disorder (ZAN-BPD): A continuous measure of DSM-IV borderline psychopathology. Journal of Personality Disorders. 2003; 17:233242. [PubMed: 12839102]

Zanarini MC. Psychotherapy of borderline personality disorder. Acta Psychiatrica Scandinavica. 2009; 120:373-377. [PubMed: 19807718]

Zanarini MC, Frankenburg FR, Hennen J, Reich DB, Silk KR. Prediction of the 10-year course of borderline personality disorder. The American Journal of Psychiatry. 2006; 163:827-832. [PubMed: 16648323]

Zanarini, MC.; Frankenburg, FR.; Sickel, AE.; Young, L. Diagnostic interview for DSM-IV personality disorders. Boston, MA: McLean Hospital; 1996.

Zanarini MC, Frankenburg FR, Vujanovic AA, Hennen J, Reich DB, Silk KR. Axis II comorbidity of borderline personality disorder: Description of 6-year course and prediction to time-to-remission. Acta Psychiatrica Scandinavica. 2004; 110:416-420. [PubMed: 15521825]

Zanarini MC, Skodol AE, Bender D, Dolan R, Sanislow C, Schaefer E, Gunderson JG. The collaborative longitudinal personality disorders study: Reliability of axis I and II diagnoses. Journal of Personality Disorders. 2000; 14:291-299. [PubMed: 11213787] 


\section{Table 1}

Pretreatment Demographic, Clinical, and Diagnostic Data for Intent-to-Treat Sample $(\mathrm{N}=51)$

\begin{tabular}{|c|c|}
\hline \multicolumn{2}{|l|}{ Demographic Characteristics } \\
\hline Age & mean $=32.5 \pm 10.9$ \\
\hline Racial/ethnic minority & $19.6 \%(\mathrm{n}=10)$ \\
\hline Lesbian/bisexual/questioning & $16.0 \%(\mathrm{n}=8)$ \\
\hline \multicolumn{2}{|l|}{ Marital status: } \\
\hline Single & $56.9 \%(\mathrm{n}=29)$ \\
\hline Married & $21.6 \%(\mathrm{n}=11)$ \\
\hline Separated/Divorced & $21.6 \%(\mathrm{n}=11)$ \\
\hline \multicolumn{2}{|l|}{ Highest educational attainment } \\
\hline High school graduate & $62.7 \%(\mathrm{n}=32)$ \\
\hline College graduate & $23.5 \%(\mathrm{n}=12)$ \\
\hline \multicolumn{2}{|l|}{ Income } \\
\hline$<\$ 20,000$ & $42 . .9 \%(n=21)$ \\
\hline$\$ 20,000-\$ 59,999$ & $34.7 \%(\mathrm{n}=17)$ \\
\hline$>\$ 60,000$ & $22.4 \%(\mathrm{n}=11)$ \\
\hline \multicolumn{2}{|l|}{ Clinical Characteristics } \\
\hline Number of BPD criteria (DIPD-IV) & mean $=6.2(\mathrm{SD}=1.7)$ \\
\hline$\%$ meeting full criteria for BPD & $86.3 \%(\mathrm{n}=44)$ \\
\hline Suicide attempt in lifetime & $58.8 \%(\mathrm{n}=30) ;$ range $=0-16$ \\
\hline Suicide attempt past year & $17.6 \%(\mathrm{n}=9) ;$ range $=0-2$ \\
\hline DSH frequency in past 3 mos. & Mean $=30.4 \pm 57.9$ \\
\hline Inpatient hospitalization past year & $19.6 \%(\mathrm{n}=10)$ \\
\hline Total hours/week of ongoing therapy & mean $=1.6(\mathrm{SD}=2.0)$ \\
\hline Hours/week individual therapy & mean $=0.8(\mathrm{SD}=0.6)$ \\
\hline Hours/week group therapy & mean $=0.3(\mathrm{SD}=1.0)$ \\
\hline Number psychiatric medications & mean $=1.8(\mathrm{SD}=1.5)$ \\
\hline Number mos. with individual therapist & mean $=15.5(\mathrm{SD}=21.1)$ \\
\hline \multicolumn{2}{|l|}{ Diagnostic Data } \\
\hline \multicolumn{2}{|l|}{ Lifetime Axis I disorders } \\
\hline Major depression & $76.5 \%(\mathrm{n}=39)$ \\
\hline Drug dependence & $25.5 \%(\mathrm{n}=13)$ \\
\hline Alcohol dependence & $25.5 \%(\mathrm{n}=13)$ \\
\hline Anxiety disorder & $78.4 \%(\mathrm{n}=40)$ \\
\hline Posttraumatic Stress Disorder & $51.0 \%(\mathrm{n}=26)$ \\
\hline Panic Disorder & $29.4 \%(\mathrm{n}=15)$ \\
\hline Generalized Anxiety Disorder & $19.6 \%(\mathrm{n}=10)$ \\
\hline Social Phobia & $25.5 \%(\mathrm{n}=13)$ \\
\hline \multicolumn{2}{|l|}{ Current Axis I disorders } \\
\hline Major depression & $41.2 \%(\mathrm{n}=21)$ \\
\hline Anxiety disorder & $58.8 \%(\mathrm{n}=30)$ \\
\hline
\end{tabular}




\begin{tabular}{lc} 
Posttraumatic Stress Disorder & $31.4 \%(\mathrm{n}=16)$ \\
Panic Disorder & $21.6 \%(\mathrm{n}=11)$ \\
Generalized Anxiety Disorder & $17.6 \%(\mathrm{n}=9)$ \\
Social Phobia & $21.6 \%(\mathrm{n}=11)$ \\
Axis II comorbidity & $39.2 \%(\mathrm{n}=20)$ \\
Cluster A PD & $5.9 \%(\mathrm{n}=3)$ \\
Cluster B PD (other than BPD) & $15.7 \%(\mathrm{n}=8)$ \\
Cluster C PD & $33.3 \%(\mathrm{n}=17)$ \\
\hline
\end{tabular}




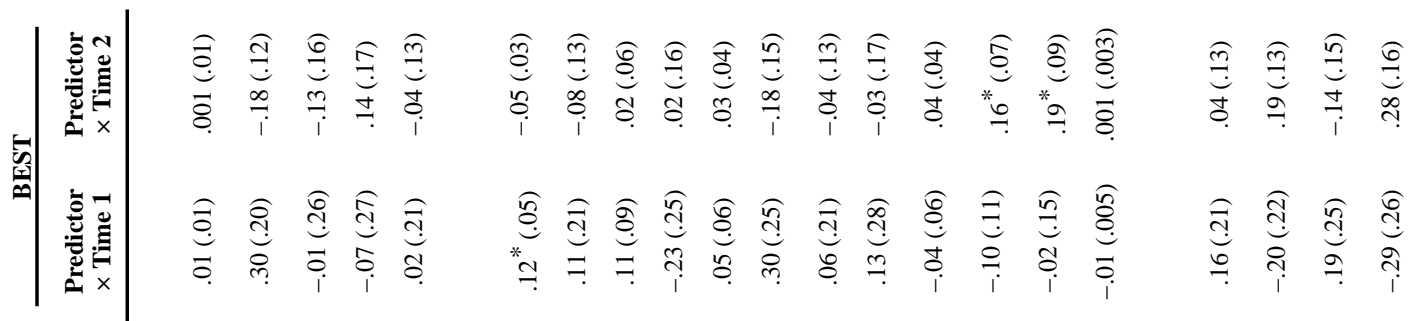

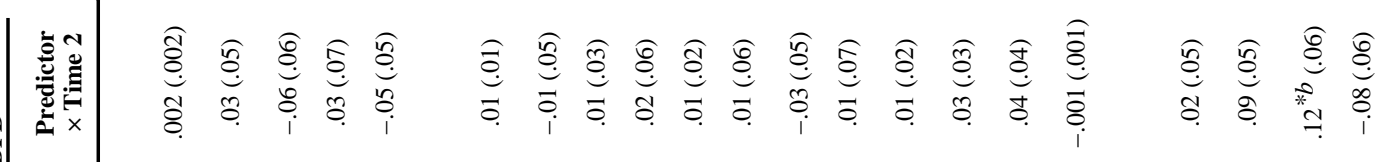

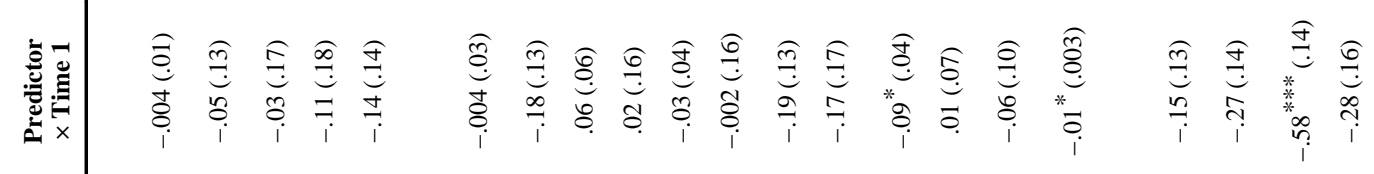

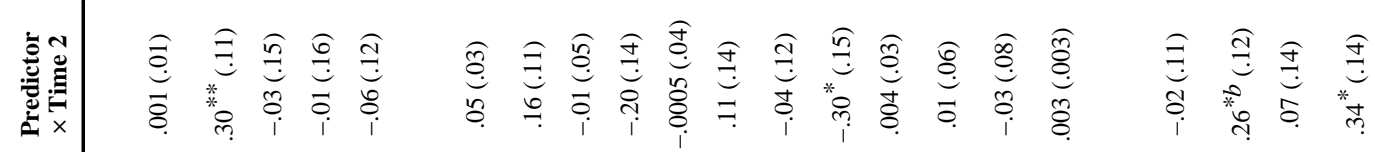

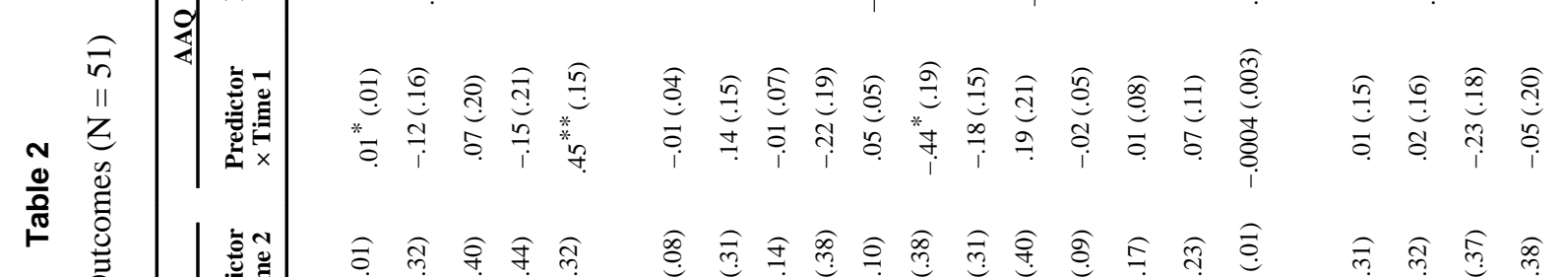

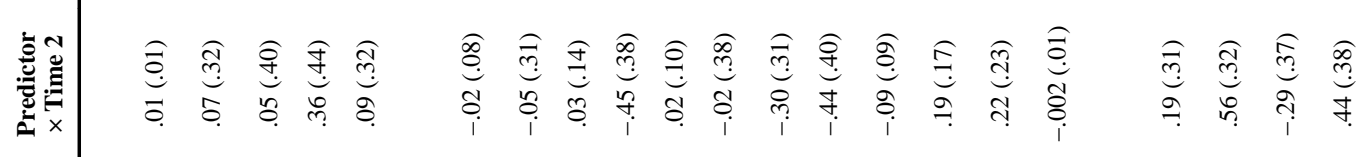

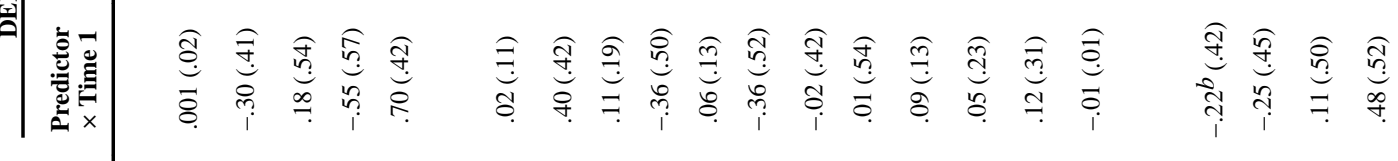

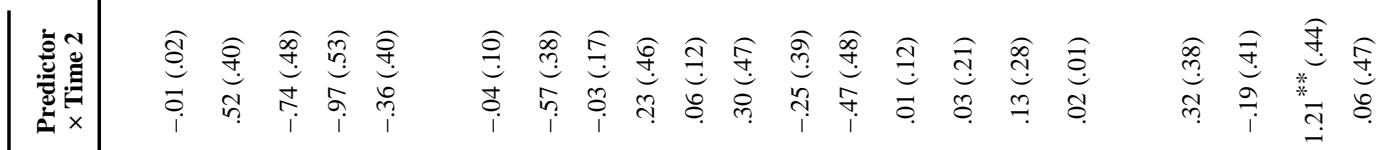

到

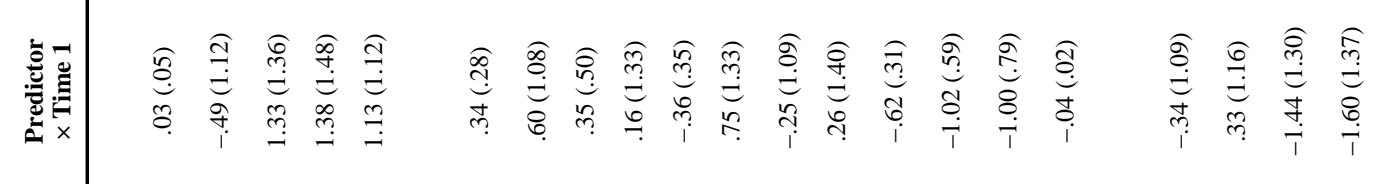

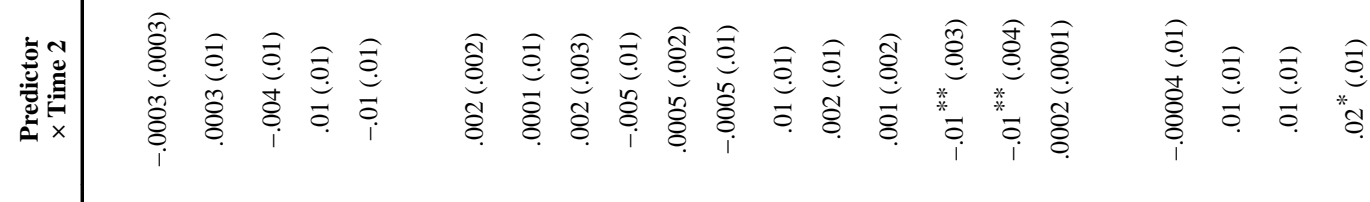

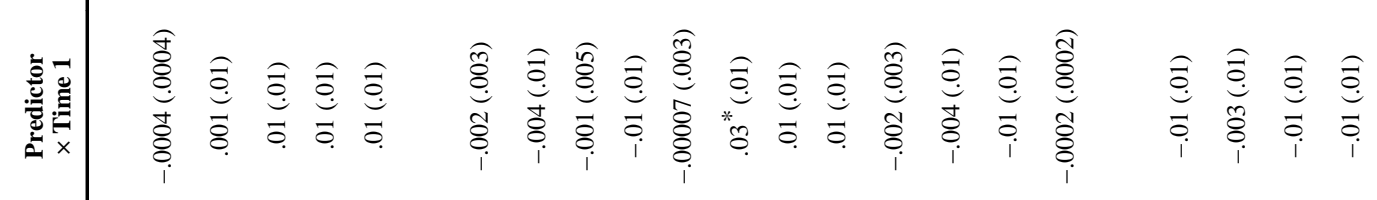

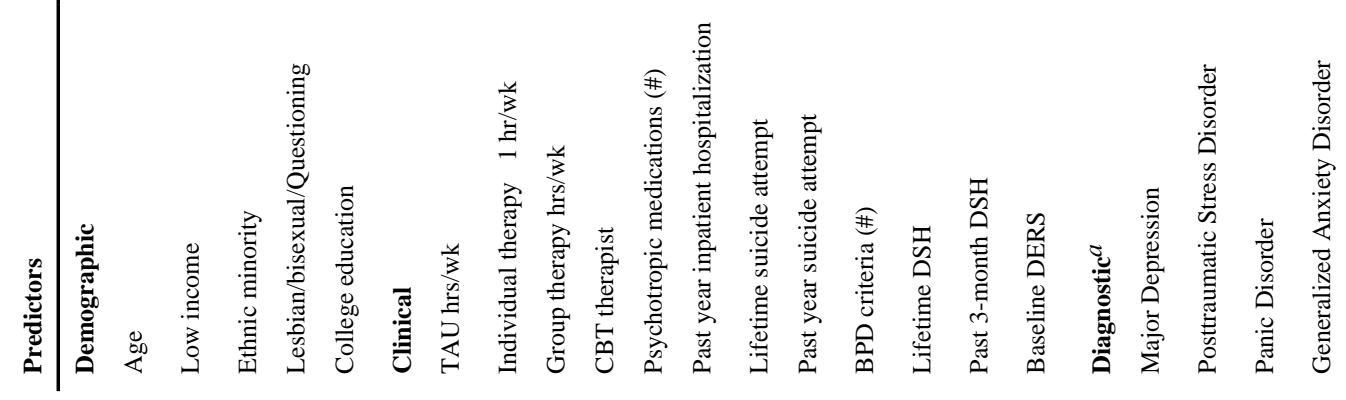


